Gutemberg Bruno da Silva

Colorimetria - Propagação de Erros e Cálculo da Incerteza de Medição nos Resultados Espectrofotométricos

DISSERTAÇÃO DE MESTRADO

Programa de Pós-Graduação em Metrologia 


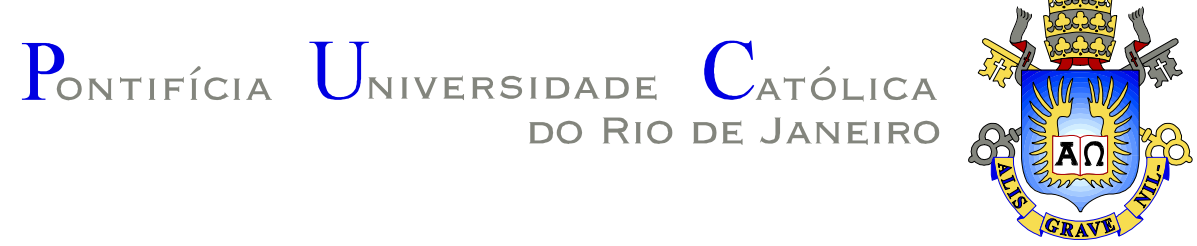

Gutemberg Bruno da Silva

Colorimetria - Propagação de Erros e Cálculo da Incerteza de Medição nos Resultados Espectrofotométricos

Dissertação apresentada ao Programa de PósGraduação em Metrologia da PUC-Rio como requisito parcial para obtenção do título de Mestre em Metrologia.

Orientador: Prof. Robert Hirschler

Co-orientador: Prof. Maurício Nogueira Frota

Rio de Janeiro

Março de 2004 


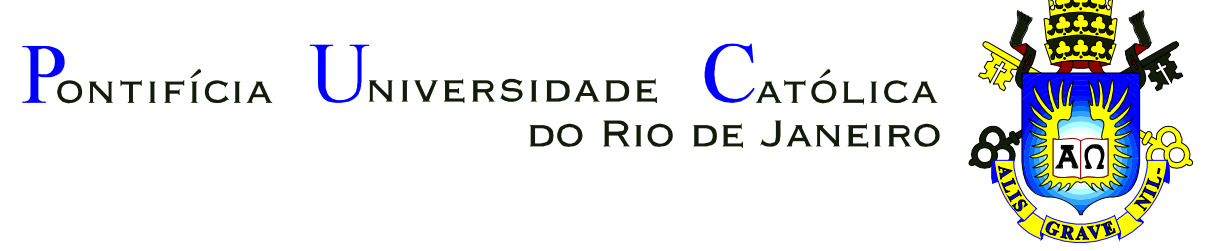

Gutemberg Bruno da Silva

\section{Colorimetria - Propagação de Erros e Cálculo da Incerteza de Medição nos Resultados Espectrofotométricos}

Dissertação apresentada como requisito parcial para obtenção do grau de Mestre pelo Programa de Pós-graduação em Metrologia do Centro Técnico Científico da PUC-Rio. Aprovada pela Comissão Examinadora abaixo assinada.

Prof. Robert Hirschler Orientador Centro de Tecnologia da Indústria Química e Têxtil SENAI/CETIQT

Prof ${ }^{\mathrm{a}}$. Elisabeth Costa Monteiro Programa de Pós-Graduação em Metrologia Pontifícia Universidade Católica do Rio de Janeiro - PUC-Rio

Prof ${ }^{a}$ Paula Medeiros Proença de Gouvêa Programa de Pós-Graduação em Metrologia Pontifícia Universidade Católica do Rio de Janeiro - PUC-Rio

Prof ${ }^{\mathrm{a}}$ Danays Morejón González Departamento de Física - PUC-RIO Pontifícia Universidade Católica do Rio de Janeiro - PUC-Rio

Prof. José Eugenio Leal

Coordenador Setorial do Centro Técnico Científico - PUC-Rio

Rio de Janeiro, 22 de março de 2004 
Todos os direitos reservados. É proibida a reprodução total ou parcial do trabalho sem autorização da universidade, do autor e do orientador.

\section{Gutemberg Bruno da Silva}

Graduou-se em Engenharia Operacional Mecânica na Escola Naval em 1978. Pós-graduou-se em Pesquisa Operacional em 1987 na Universidade Federal Fluminense. Participou de diversos congressos promovidos pela Sociedade Brasileira de Pesquisa Operacional (SOBRAPO) e de Encontros Nacionais de Engenharia de Produção (ENEGEP). Realizou vários cursos na área de Metrologia e de Sistemas da Qualidade. Trabalha atualmente na área de Avaliação Operacional de Sistemas, no Centro de Análises de Sistemas Navais.

Ficha Catalográfica

Silva, Gutemberg Bruno

Colorimetria - propagação de erros e cálculo da incerteza de medição em resultados espectrofotométricos / Gutemberg Bruno da Silva; orientador: Robert Hirschler; co-orientador: Maurício Nogueira Frota. Rio de Janeiro: PUC-Rio, Programa de PósGraduação em Metrologia, 2004.

93 f.: il. ;30 cm

Dissertação (mestrado) - Pontifícia Universidade Católica do Rio de Janeiro, Programa de Pós-Graduação em Metrologia para a Qualidade Industrial.

Inclui referências bibliográficas.

1. Metrologia - Teses. 2. Colorimetria. 3. Incerteza de medição. 4. Propagação de erro. 5 Refletância. 6. Correlação. I Hirschler, Robert. II. Frota, Maurício Nogueira. III. Pontifícia Universidade Católica do Rio de Janeiro. Programa de Pós-Graduação em Metrologia para a Qualidade Industrial. IV. Título 


\section{Agradecimentos}

Para a consecução desta dissertação, contei com a inestimável ajuda de algumas instituições e pessoas que me agraciaram com o resultado dos seus esforços. Dessa forma, sou grato:

À PUC-Rio, pela excelência do ambiente universitário, proporcionando acesso a um programa de pós-graduação reconhecido nacional e internacionalmente.

Ao Dr. Robert Hirschler, pesquisador do SENAI/CETIQT, pelo fornecimento do material utilizado na pesquisa, pela orientação segura por áreas do conhecimento que eu ainda não tinha visitado e pela amizade sincera.

Ao professor Maurício Frota, Coordenador do Programa de Pós-graduação em Metrologia da PUC-Rio, pela co-orientação e incentivo permanente.

À Financiadora de Estudos e Projetos (FINEP) e ao Ministério da Ciência e Tecnologia (MCT), pelo apoio institucional do Fundo Setorial Verde-Amarelo (Convênio FIEP/MCT Convênio FINEP 22.01.0692.00, Referência 1974/01) que permitiu enquadrar a presente pesquisa de Mestrado dentre os dez projetos-piloto que direcionaram o esforço acadêmico de pesquisa do Programa de Pós-graduação em Metrologia da PUC-Rio para a solução de problemas industriais cujas soluções requerem conhecimentos avançados em metrologia. Ainda nesse contexto, agradecimentos são também devidos ao SENAI/CETIQT, que não apenas identificou o tema da pesquisa junto às necessidades do setor têxtil, mas ofereceu sua excelente infra-estrutura laboratorial em metrologia da cor para equacioná-lo.

Aos membros da banca examinadora da dissertação, professora Elisabeth Costa Monteiro, professora Paula Medeiros Proença de Gouvêa e professora Danays Morejón González, pela atenção com que examinaram o meu trabalho.

Ao amigo Maurício José Machado Guedes, pela compreensão e incentivo.

Ao colega José Correa Paes Filho, pela orientação segura sobre programas de simulação.

Ao colega Fernando Ayres, pela indicação e orientação acerca da utilização do programa Maple de cálculos matemáticos, e

Às colegas Cristiane Borges de Almeida Silva e Cássia Cristina de Melo, respectivamente assistente técnica e aluna do CETIQT, pelo fornecimento dos preciosos dados que possibilitaram o desenvolvimento da minha pesquisa. 


\section{Resumo}

Silva, Gutemberg Bruno; Hirschler, Robert. Colorimetria - Propagação de erros e cálculo da incerteza de medição nos resultados espectrofotométricos. Rio de Janeiro, 2004. 93 p. Dissertação de Mestrado, Programa de Pós-Graduação em Metrologia, Pontifícia Universidade Católica do Rio de Janeiro.

\section{Colorimetria - Propagação de erros e cálculo da incerteza de medição} nos resultados espectrofotométricos trata da medição da cor de objetos, baseada nas medições de irradiância espectral (objetos luminosos) ou de refletância ou transmitância espectral (objetos opacos ou transparentes), seguidas por cálculos colorimétricos conforme o sistema proposto pela CIE. As medições são normalmente feitas em intervalos de $5 \mathrm{~nm}$ (ou $10 \mathrm{~nm}$ ) na faixa espectral de 360 a 780nm, e os três valores triestímulos (X, Y e Z) são calculados usando-se 42-84 pontos medidos por equações padrões.

A distribuição dos valores medidos $\mathrm{R}_{\lambda}$ é, provavelmente, normal, com uma correlação entre os valores obtidos variável em posições diferentes do espectro. As distribuições dos valores e as correlações entre $\mathrm{X}, \mathrm{Y}$ e Z são desconhecidas e dependem da forma da curva espectral da cor e do funcionamento dos instrumentos de medição.

No controle instrumental das cores são usadas fórmulas muito complexas, baseadas nas transformações não lineares dos valores $\mathrm{X}, \mathrm{Y}$ e Z em $L^{*}, a^{*}, b^{*}, C^{*}$ e $h^{\circ}$. A determinação da incerteza dos resultados dados em coordenadas CIELAB ou expressos em fórmulas de diferenças $\Delta E^{*}, \Delta E_{C M C}$ ou CIE $\Delta E 2000$ é fundamental no controle instrumental das cores em qualquer indústria. À base de um número elevado de medições repetidas de várias amostras têxteis e padrões cerâmicos, são analisadas a distribuição e outras características estatísticas dos valores $\mathrm{R}_{\lambda}$ diretamente medidos e, usando o método de propagação de erros, são calculadas as incertezas das medições em termos colorimétricos.

A pesquisa de mestrado objeto do presente trabalho desenvolveu-se sob a égide de um convênio de cooperação celebrado entre o Programa de Pósgraduação em Metrologia da PUC-Rio e o SENAI/CETIQT, viabilizado a 
inclusão dessa pesquisa dentre os dez projetos-piloto que participaram do Convênio FINEP/MCT nº 22.01.0692.00, Referência 1974/01, que aportou recursos do Fundo Setorial Verde Amarelo para direcionar o esforço de pesquisa em metrologia para a solução de um problema de interesse do setor têxtil que fez uso de conhecimentos avançados de metrologia da cor. Relacionado à demanda de medições espectrofotométricas com rigoroso controle metrológico, o desenvolvimento e a orientação acadêmico-científica da presente dissertação de mestrado deu-se nas instalações do SENAI/CETIQT, que possui comprovada competência técnica e científica na área e uma adequada infra-estrutura laboratorial em metrologia da cor de suporte ao trabalho.

\section{Palavras-chave}

Metrologia; Colorimetria; Incerteza de Medição; Propagação de Erro; Refletância; Correlação. 


\section{Abstract}

Silva, Gutemberg Bruno; Hirschler, Robert (Advisor). Colorimetry Propagation of Errors and Uncertainty Calculations in Spectrophotometric Measurements. Rio de Janeiro, 2004. 93 p. MSc. Dissertation - Programa de Pós-Graduação em Metrologia, Pontifícia Universidade Católica do Rio de Janeiro.

\section{Colorimetry - Propagation of Errors and Uncertainty Calculations in} Spectrophotometric Measurements treats the measurement of the colour of objects, based on the measurement of spectral irradiance (self-luminous objects) or that of spectral reflectance or transmittance (opaque or transparent objects), followed by colorimetric calculations according to the CIE system. Measurements are generally made in 5nm (or $10 \mathrm{~nm}$ ) intervals in the spectral range of 360 to 780 $\mathrm{nm}$, and the 3 tristimulus values (X, $\mathrm{Y}$ and $\mathrm{Z}$ ) are calculated from the 42-84 measurement points by standard equations.

The statistical distribution of the measured $\mathrm{R}_{\lambda}$ values is probably normal; the correlation between the values varies depending on their position in the spectrum. The distribution of and the correlation between the $\mathrm{X}, \mathrm{Y}$ and $\mathrm{Z}$ values are not known and they depend on the form of the spectral curve of each colour and on the operation of the measuring instrument.

Complex formulae are used in the instrumental control of colours based on non-linear transformations of the $X, Y$ and $Z$ values into $L^{*} a * b * C * h{ }^{\circ}$. The determination of the uncertainty of the results given in CIELAB coordinates or expressed in one of the colour difference formulae $\Delta \mathrm{E}^{*}, \Delta \mathrm{E}_{\mathrm{CMC}}$ or CIE $\Delta \mathrm{E} 2000$ is fundamental in the instrumental control of colours in any industry. Based on a large number of repeated measurements of different textile samples and ceramic standards, the distribution and other statistical characteristics of the directly measured $\mathrm{R}_{\lambda}$ values are analysed and - using the propagation of errors method the uncertainties are calculated in colorimetric terms.

The present research, a M. Sc. Dissertation work, was developed under the auspices of a co-operation agreement celebrated between the Post-graduate Programme in Metrology of PUC-Rio and SENAI/CETIQT, allowing for the inclusion of this M.Sc. Dissertation among the ten pilot projects which benefited 
from the financial support received from the FINEP/MCT Agreement $\mathrm{n}^{\mathrm{o}}$ 22.01.0692.00, Reference 1974/01 (Fundo Verde-Amarelo). The project aims at driving the research effort in metrology to the solution of industrial problems, in this case the solution of a problem identified within the textile sector which requires to its solution advanced knowledge of colour metrology.

Related the spectrophotometer measurements under the highest level of metrological control, the development and academic-scientific supervision of this M. Sc. Dissertation was performed at the laboratory facility of SENAI/CETIQT, an institution with proven technical and scientific competence in the field having sophisticated and well equipped laboratories in colour metrology meeting the measurement requirements needed to support the development of this research.

\section{Keywords}

Metrology; Colorimetry; Uncertainty in Measurement; Error Propagation; Factor of Reflectance; Correlation. 


\section{Sumário}

1 INTRODUÇÃO 15

1.1. Propósito 15

1.2. Organização da Dissertação 16

2 Fundamentos Teóricos - Revisão Bibliográfica 17

2.1. Conceitos Básicos de Colorimetria 17

$\begin{array}{ll}\text { 2.1.1. A Cor } & 17\end{array}$

2.1.2. Definições 18

2.1.3. Fatores Fundamentais 19

2.1.4. Atributos Básicos da Cor 19

2.1.5. Sistemas de Ordenação de Cor 20

2.1.6. Combinação de Luzes ou Pigmentos e Filtros Coloridos 22

2.1.7. O Espectrofotômetro 23

2.1.8. O Observador Padrão 26

2.1.9. Valores Espectrais Triestímulos 27

2.1.10. O lluminante Padrão 28

2.1.11. As Cores em Números - O Espaço CIELAB 29

2.1.12. Tolerâncias Industriais 33

2.2. Interação da Luz com Materiais Opacos 35

2.2.1. A Teoria de Kubelka-Munk 35

2.2.2. Refletância e Concentração de Substratos Têxteis 36

2.3. Incerteza de Medição e Repetitividade de Tingimentos Têxteis 38

2.4. Ferramentas Estatísticas na Colorimetria 42

2.4.1. Organização dos Dados - Estatística Descritiva 42

2.4.2. A Distribuição Amostral de $\bar{X}$ e de S 46

2.4.3. Regiões de Confiança e Comparações Simultâneas de Médias 47

2.4.4. Elipses de Confiança 50

3 Calibração de Padrões de Transmitância e Refletância - Bibliografia

$\begin{array}{ll}\text { Comentada } & 53\end{array}$ 
3.1. Livros $\quad 54$

3.2. Artigos 55

3.2.1. Estatística e Cálculo Matricial 55

3.2.2. Erro Instrumental $\quad 57$

3.2.3. Função de Matização de Cor 62

3.2.4. Formulação de Receitas 63

3.2.5. Correlação 65

4. Trabalho Desenvolvido 69

4.1. Incerteza das Medições Espectrofotométricas 69

4.2. Propagação de Erros nas Medições Espectrofotométricas 70

4.3. Propagação de Erros nas Colorações Têxteis 74

4.4. Simulações de Monte Carlo 75

$\begin{array}{ll}\text { 4.5. Resultados e Discussão } & 77\end{array}$

5 Conclusões e Recomendações 85

6 Referências Bibliográficas $\quad 87$

$\begin{array}{ll}7 \text { Anexo } & 93\end{array}$ 


\section{Lista de siglas utilizadas}

AATCC - American Association of Textile Chemists and Colorists.

AIC - Association Internationale de la Couleur.

ANLAB - Fórmula de diferença de cor Adams-Nickerson L, a, b.

ASTM - American Society for Testing and Materials.

CETIQT - Centro de Tecnologia da Indústria Química e Têxtil.

CIE - Comission Internacionale de l'Éclairage.

CIELAB - Espaço da cor que segue as normas da CIE e tem coordenadas cartesianas $L^{*}, a^{*}, b^{*}$ ou coordenadas polares $C^{*}, h^{0}$.

CIELUV - Espaço da cor que segue as normas da CIE e com coordenadas cartesianas $L^{*}, u^{*}, v^{*}$.

ISO GUN - International Organization for Standardization - Guide to the Expression of Uncertainty in Measurement.

JSDC - Journal of the Society of Dyers and Colourists.

$K_{\lambda}$ - Absorbância para cada comprimento de onda $\lambda$.

MCDS - Diferença média de cor do padrão.

NCS - Natural Color System. Um dos sistemas de representação de cor.

$R_{\lambda}$ - Refletância para cada valor de comprimento de onda $\lambda$.

$S_{\lambda}$ - Dispersão para cada valor de comprimento de onda $\lambda$.

SENAI - Serviço Nacional de Aprendizagem Industrial.

SPD - Spectral Power Distribution (Distribuição Espectral de Potência).

UKAS - United Kingdom Accreditation Service.

UMIST - University of Manchester Institute of Science and Technology.

UV - Raio ultra-violeta.

$\mathrm{U}^{*}, \mathrm{~V}^{*}, \mathrm{~W}^{*}$ - Espaço da cor.

$\mathrm{X}, \mathrm{Y}, \mathrm{Z}$ - Coordenadas triestímulos. São os valores provenientes da convolução entre características do iluminante, do observador padrão e da amostra.

$\mathrm{x}, \mathrm{y}, \mathrm{z}$ - Coordenadas de cromaticidade.

$\bar{x}, \bar{y}, \bar{z}$ - Funções de colorimetria.

$\Delta E_{a b}^{*}$ - Grandeza que representa a diferença de cor no espaço CIELAB. 
$\Delta E_{C M C}$ - Grandeza que representa a diferença de cor no espaço CMC.

$\Delta E_{00}$ - Grandeza que representa a diferença de cor no espaço CIE 2000. 


\section{Lista de figuras}

Figura 1 - Exemplo de atributos básicos da cor. Fonte: Hirschler (2003).

Figura 2 - Sistema Munsell. Página de tonalidade constante 5.0 B. Fonte:

Software CMC da GretagMacbeth, www.gretagmacbeth.com. 21

Figura 3 - Mistura aditiva de cores. Fonte: Hirschler (2002). 22

Figura 4 - Mistura subtrativa de cores. Fonte: Hirschler (2002). 23

Figura 5 - Diagrama esquemático do espectrofotômetro. Fonte: Berns (2000). 23

Figura 6 - Geometrias de iluminação e de observação recomendadas pela CIE.

Fonte: Judd-Wyszecki. $\quad 24$

Figura 7 - Diagrama do espectrofotômetro MINOLTA CM-3200d. Fonte:

Folheto Minolta www.minolta.com. 25

Figura 8 - Curvas de refletância espectral de amostras amarela, vermelha e azul.

Fonte: Hirschler (2003). 26

Figura 9 - O observador padrão. Fonte: Judd-Wyszecki. 26

Figura 10 - Campos visuais dos observadores. Fonte: Judd-Wyszecki. 27

Figura 11 - Valores espectrais triestímulos. Fonte: Dados da Norma CIE/ISO. 27

Figura 12 - Diferentes tipos de iluminantes. Fonte: Folheto GretagMacBeth. 29

Figura 13 - Valores triestímulos X, Y e Z. Fonte: Hirschler (2003). 29

Figura 14 - Diagrama de cromaticidade. Fonte: Software da efg’s Computer Lab.

$\begin{array}{ll}\text { www.efg2.com/lab. } & 31\end{array}$

Figura 15- O espaço CIELAB. Fonte: Folheto Minolta. www.minolta.com. 33

Figura 16 - Elipse de tolerância. Fonte: Hirschler (2003). 34

Figura 17 - Determinação do tamanho dos elipsóides da tolerância industrial.

Fonte: Hirschler (2003).

Figura 18 - O modelo simplificado de Kubelka e Munk. Fonte: Kuehni (1975). 35

Figura 19 - Elipses de aceitação (maior) e da média. Fonte: autor 52

Figura 20 - Elipsóide de aceitação. Fonte: autor 52

Figura 21 - Valores médios (curva) e desvios-padrão (multiplicado por 10) dos pontos (linhas) de três azulejos medidos 50 vezes num espectrofotômetro.

Fonte: Hirschler (2004). $\quad 80$

Figura 22 - Relação entre erro percentual e diferença de cor. Fonte: autor. 81 


\section{Lista de tabelas}

Tabela 1 - Erros na cor $\left(\Delta E_{L a b}\right)$, devido a erros na concentração de corantes. 81 Tabela 2 - Exemplo de resultados obtidos por cálculo e por simulação, para os valores triestímulos X, Y e Z e do espaço $L^{*} a^{*} b^{*}$ de amostra têxtil. 82 